\title{
Arteriovenous Malformation (AVM) Treated with Robotic Radiosurgery: Impact of Beam Reduction in 12 Gy Normal Brain Volume and It's Clinical Implication
}

\author{
Debnarayan Dutta ${ }^{*}$, Sathiya Krishnamoorthy², Ganapathy Krishnan ${ }^{3}$, \\ Harikrishnaperumal Sudahar ${ }^{3}$ \\ ${ }^{1}$ Department of Radiation Oncology, Amrita Institute of Medical Science, Kochi, India \\ ${ }^{2}$ Department of Radiation Oncology, Global Hospital, Chennai, India \\ ${ }^{3}$ Department of Medical Physics, Apollo Speciality Cancer Hospital, Chennai, India \\ Email: *duttadeb07@gmail.com
}

How to cite this paper: Dutta, D., Krishnamoorthy, S., Krishnan, G. and Sudahar, H. (2019) Arteriovenous Malformation (AVM) Treated with Robotic Radiosurgery: Impact of Beam Reduction in 12 Gy Normal Brain Volume and It's Clinical Implication. International Journal of Medical Physics, Clinical Engineering and Radiation Oncology, 8, 131-140.

https://doi.org/10.4236/ijmpcero.2019.83012

Received: June 20, 2019

Accepted: August 11, 2019

Published: August 14, 2019

Copyright $\odot 2019$ by author(s) and Scientific Research Publishing Inc. This work is licensed under the Creative Commons Attribution International License (CC BY 4.0).

http://creativecommons.org/licenses/by/4.0/

\section{(c) (i) Open Access}

\begin{abstract}
Purpose: Dosimetric study to evaluate impact of "beam" reduction in AVM radiosurgery on normal brain dose parameters and it's clinical implications. Materials and Methods: Five small volume AVMs (nidus volume 0.31 - 1.94 cc) planned for single fraction SRS with robotic radiosurgery system. Planning scans done with CT scan brain, CT \& MR angiography, then nidus volume and organ at risk (OARs) were contoured. Planning was done with multiplan planning system. Plan evaluated as per Flickenger model parameters of 12 Gy nomal brain vol \& marginal dose. $7.5 \mathrm{~mm}$ and $10 \mathrm{~mm}$ cons used, optimization done with seqential algorithm. $20 \mathrm{~Gy}$ was prescribed to isodose with appropriate nidus coverage $(>98 \%)$. Total beams of five plans were 85 - 250, monitor unit 17,259 - 24,602 MU. 12 Gy normal brain volume is $0.9-7.6 \mathrm{cc}$. Then beam reduction is done by reducing beams with minimum MU in steps of 50,100,150, 200, $250 \mathrm{MU}$ and after beam reduction, re-optimization done. Prescription isodose was changed to keep the nidus coverage $>98 \%$. Impacts of beam reduction on 12 Gy normal brain vol and conformity/homogeniety index were analyzed. Results: Optimal plans of five patients with $20 \mathrm{~Gy}$ prescribed to $88 \%$ - $90 \%$ isodose, nidus coverage more than $98 \%$. In dosimetric parameters, mean CI was $1.36-1.51$, nCI $1.41-1.51$, HI 1.1 - 1.4 and mean 12 Gy normal brain volume 0.17, 1.44, 5.3, 5.5 and $7.6 \mathrm{cc}$ respectively. After beam reduction of less than $50 \mathrm{MU}$ contribution (in case\#1), prescibing at suitable isodose (85\%) beam reduces to 79 and $12 \mathrm{~Gy}$ volume marginally increases to $26.4 \mathrm{cc}$. Beam reduction of less than $100 \mathrm{MU}$ reduces to 53 - 92 beamlets. Reduction of beams with less than 150 MU con-
\end{abstract}


tribution did not significantly change the $12 \mathrm{~Gy}$ normal brain volume. However, reduction of beamlets with more than $200 \mathrm{MU}, 250 \mathrm{MU}, 300 \mathrm{MU}, 450$ $\mathrm{MU}$ and $550 \mathrm{MU}$ significantly affects the $12 \mathrm{~Gy}$ normal brain volume. Prescription-isodose modified from $83 \%$ to $50 \%$ to have $>98 \%$ coverage. CI and $\mathrm{HI}$ increased from $1.36-1.51$ to $2.51-2.63$ and $1.1-1.4$ to $1.52-1.54$ respectively. There was exponential increase in 12 Gy volume with reduction of beams with higher proportion in larger nidus. Conclusions: In robotic radiosurgery system, beam reduction even after re-optimization impairs the conformity index and increase 12 Gy normal brain volume, hence long-term toxicity. Optimal beam numbers are required for optimal plan generation.

\section{Keywords}

AVM, Robotic Radiosurgery, CK-Cyberknife, 12 Gy Volume, Beam Reduction

\section{Introduction}

Arteriovenous malformations (AVMs) of the brain are genetically determined abnormal formation of vasculature in the development which in turn may lead to a focal arterio-venous shunt and hence higher probability of rupture and bleeding [1] [2]. Patients usually present with symptoms like neurological deficits, seizures or headache. Life-time risk of hemorrhage in AVM is around $40 \%$ and the risk of haemorrhage increases annually by $2 \%$ - 3\% [1] [2]. Surgical excision is the treatment of choice in "safe" non-eloquent, superficial AVMs with high cure rate. Single fraction radio surgery (SRS) is an effective alternative especially in deep seated and AVMs located in eloquent areas of brain [3] [4] [5]. Obliteration with SRS depending upon the size of the nidus, it's location and "marginal" dose delivered [4] [5]. Usually complete obliteration rate (cure) in suitable cases varies between 70\% - 90\% [5] [6] [7]. The side effect (persistent neurological deficit) depends upon the 12 Gy normal brain volume and site of the nidus [4]. Modernprecise techniques such as multiple arcs or multiple beams (robotic radiosurgery) promise higher conformity, lesser dose to normal brain (lesser 12 Gy volume) and hence are supposed to be more effective in "border line" size nidus. However, with increase in number of beams, there is increase in monitor unit and treatment time [8] [9].

In the present study, we are analyzing the impact of reduction of beams on dosimetric parameters in optimal AVM nidus $(<2.5 \mathrm{~cm}$ nidus) planned optimally with robotic radiosurgery system. The main objective of the present study is to evaluate the impact of beam reduction on an optimal plan after re-optimization.

\section{Materials and Methods}

Five AVM patients with small volume in different part of the brain were considered for the study. All these symptomatic AVM patients (presented mostly with 
occational episodes of convulsion or headache without any obvious bleeding) were confirmed by digital subtraction angiography (DSA) and were planned for SRS with robotic radiosurgery system. Planning scans done as per the protocol with plain CT scan brain, CT angiography and MR angiography. After fusion of the images in "Multiplan" system and assessment of diagnostic DSA images, the nidus volume and organ at risk (OARs) were contoured. No PTV margin was generated in the nidus (target) and normal brain volume contoured without excluding the nidus volume. Robotic Radiosurgery (CyberKnife ${ }^{\circledR}$ ) treatment plans were generated in Multiplan treatment planning algorithm. Computed tomography images of the patients acquired with $1 \mathrm{~mm}$ spacing were used to create treatment plans. Treatment plans are generated using the sequential optimization method. Radiosurgery plans were generated with treatment paths that control the beam orientations specific to different anatomical sites. Treatment plans for patients with AVM were generated using the head path with 6D skull tracking method to deliver a dose of $20 \mathrm{~Gy}$ marginal dose in a single treatment session. Multiple shells with asymmetric margins around the target were used to limit the dose to critical structures near the target. The dose distribution in the computed tomography images of the patient's head was calculated using the "Raytracing" algorithm. Collimator sizes of 7.5 and $10 \mathrm{~mm}$ were used in the treatment plan. Number of beams, monitor unit (MU), nodes, CI, nCI, HI and 12 Gy normal brain volumes in all the plans were documented in Table 1. Approved plan had nidus coverage more than $98 \%$, optimal number of beams, acceptable $\mathrm{CI}, \mathrm{nCI}$ and $\mathrm{HI}$ values and adjacent critical structure dose within normal limits. The nidus volumes were small, ranging from $0.32 \mathrm{cc}$ to $9.4 \mathrm{cc}$ [Table $1]$. As the nidus volumes were very small and irregular in shape; approved plan $\mathrm{CI}$ and $\mathrm{HI}$ values were on the higher side (CI range 1.36 - 1.51). Plan evaluation was done as per the Flickenger model parameters of 12 Gy nomal brain volume, appropriate marginal dose with nidus coverage more than $98 \% .12$ Gy volume considered without substraction of nidus volume. As per the flickenger model, probability of persistent neurological deficits were less than $5 \%$ in all the cases Marginal dose of 20 Gy provides complete obliteration rate of $85 \%$ at 2 year. Subsequently for this study, treatment plans were generated by removing the beams with certain number of monitor units and optimized once again to meet the dose volume objectives of the treatment plan. After approval of the plan, beam reduction done in steps by reducing beams with contribution less than MU of 50,100,150, 200 and 250 respectively. The collimator size was not changed to keep uniformity and evaluate the impact of beam reduction with re-optimization. Prescription of the isodose was changed to keep nidus coverage $>98 \%$. Impact of beam reduction on the 12 Gy normal brain volume and conformity/homogeneity index was analyzed [Figure 1]. The formulae used to calculate the conformity index and the homogeneity index are given as follows. Conformity Index $(\mathrm{CI})=\left(\mathrm{V}_{\mathrm{RI}} \times \mathrm{TV}\right) /\left(\mathrm{TV}_{\mathrm{RI}}\right)^{2}$. Where $\mathrm{V}_{\mathrm{RI}}$ is the overall volume including the target volume, receiving the prescription isodose or more, TV is 
Table 1. Dosimetric parameters of plans with "beam reduction" from optimal plan of five different patients with AVM in different location of brain.

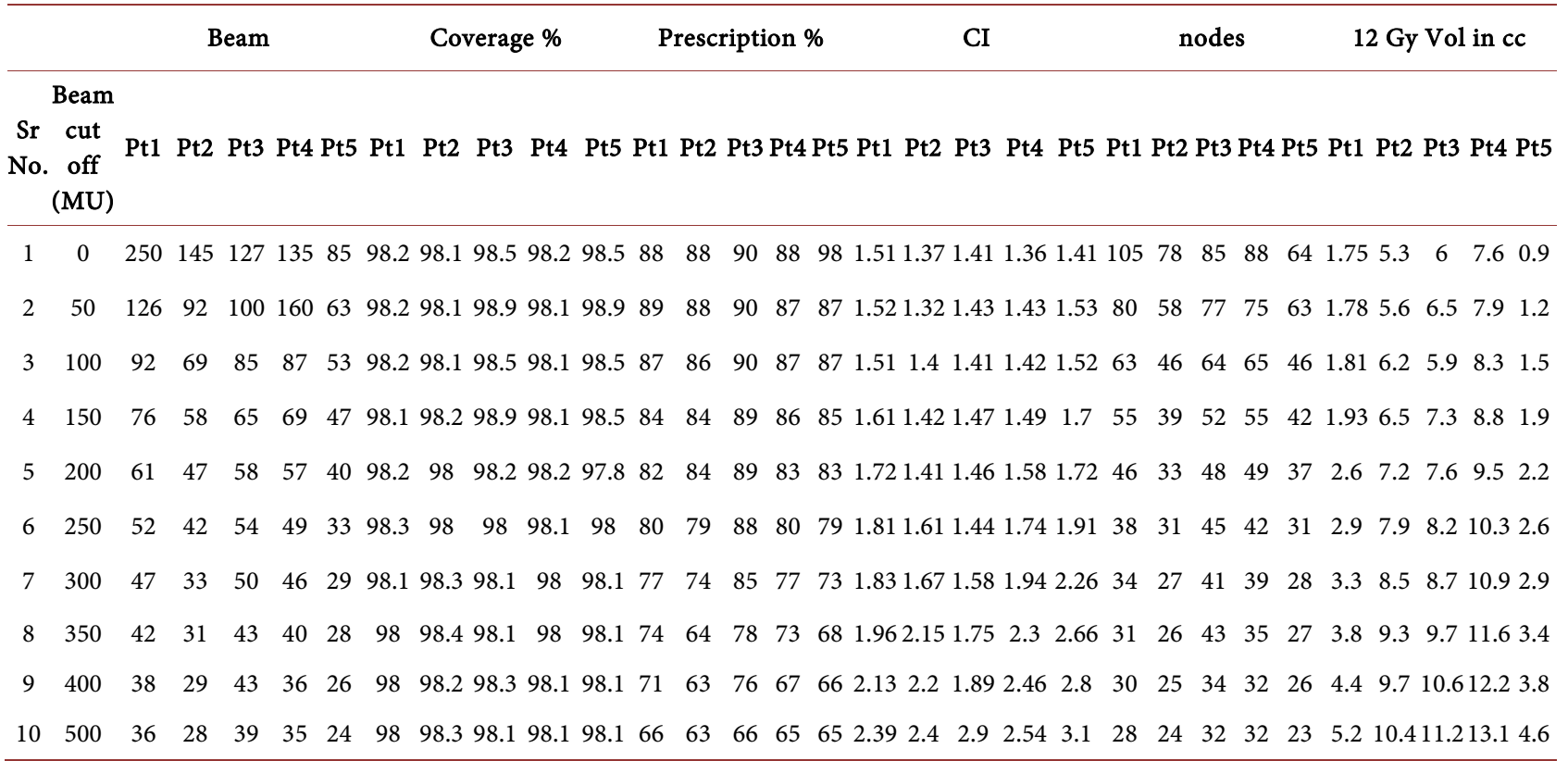

Beam reduction done with reduction of beam from optimal plan with cutoff of beam less than $50 \mathrm{MU}$, then $100 \mathrm{MU}$ and similar pattern. Re-optimization of plans were done after each beam reduction. Plan considered optimal after beam reduction and optimization with change in prescription iso-dose. Acceptable plan need to have more than $98 \%$ nidus coverage. Change in number of beamlets, nodes and $12 \mathrm{~Gy}$ normal brain volume was considered for analysis and correlation. Pt: Patient; Nidus volume: Pt1: 1.53 cc, Pt2: 1.94 cc, Pt3: 1.54 cc, Pt4: 1.86 cc, Pt5: 0.32 cc. Site: Pt1: Left Frontal lobe, Pt2: Left Frontal lobe, Pt3: Right Tempero-parietal lobe, Pt4: Left Frontal lobe, Pt5: Left Temporal lobe.

\section{Conformity \& Homogeneity Index with beam reduction}

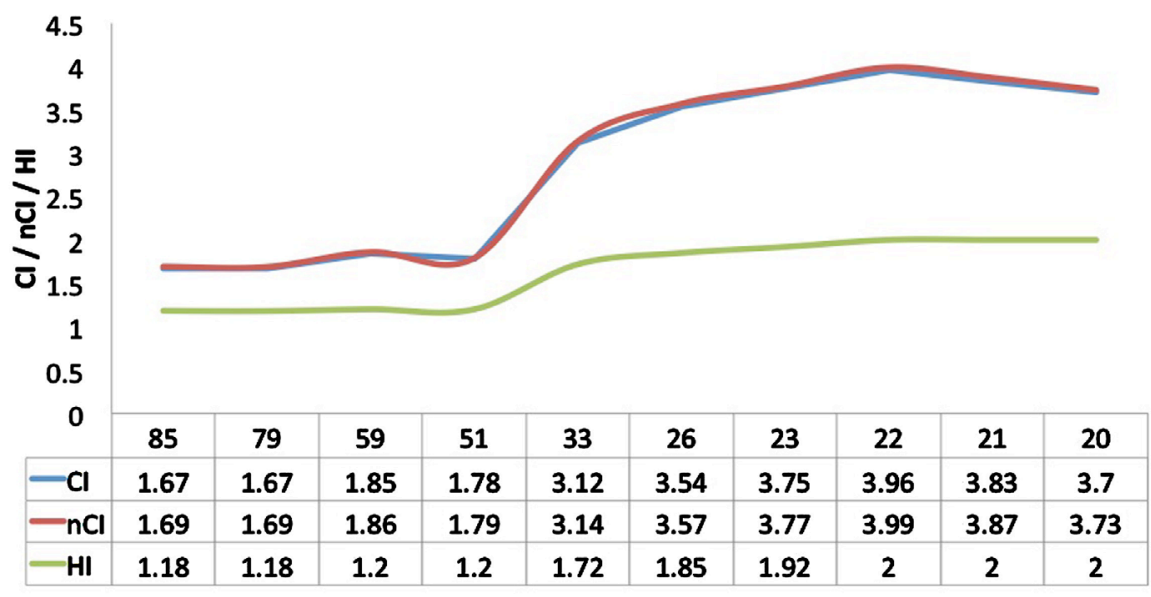

Impact of beam reduction on conformity index parameters of patient no 1 . With reduction of beam \& re-optimization, there is impairment of $\mathrm{Cl} \& \mathrm{HI}$. Cl \& $\mathrm{HI}$ impairment was dramatic after gross reduction of beams suggest that there is a threshold of beam reduction. After the threshold, gross impairment of conformity index in evident

Figure 1. Correlation of number of beamlets and 12Gy normal brain volume.

the volume of the nidus, and $\mathrm{TV}_{\mathrm{RI}}$ is the volume of the target which receives the prescription isodose or more. Homogeneity index $(\mathrm{HI})=\mathrm{D}_{\max } / \mathrm{D}_{\mathrm{RI}}$ where $\mathrm{D}_{\max }$ is the maximum dose in the target and $\mathrm{D}_{\mathrm{RI}}$ is the reference/prescription isodose [10] [11] [12]. 


\section{Results}

Optimal plans with 20 Gy marginal dose prescribed to appropriate isodose with nidus coverage more than $98 \%$ and optimal beams were described in Table 1. After reduction of beams with less than $50 \mathrm{MU}$ contribution, optimization and prescibing at suitable isodose to have nidus coverage more than $98 \%$ and then 12 Gy normal brain volumes were calculated in all the plans. Similarly further reducion of beams with contribution more than $100 \mathrm{MU}$, then 150, 200, 250 and whereon were evaluated, re-optimization done; prescription isodose changed to have nidus coverage more than $98 \%$ and then 12 Gy volumes were calculated. In all the plans, after reduction of beams more than $200 \mathrm{MU}, 250 \mathrm{MU}, 300 \mathrm{MU}, 350$ MU, 450 MU and 550 MU there was significant increase in 12 Gy normal brain volumes. In order to have nidus coverage more than $98 \%$, prescription isodose was modified from $83 \%$ to $50 \%$. CI and HI were increased respectively [Figure 2].

Correlation between the reduction of beams and increase in 12 Gy normal brain volume was done by Pearsons Correlation test (correlation 95.6\%, p-value = 0.003) [Figure 1 and Figure 2]. There was no increase in 12 Gy volume despite reduction of beams till a threshold was reached. After that there was exponential increase in 12 Gy normal brain volume with reduction of beams. It does suggest that there is a optimal scope of reduction of beams in optimal plans without increasing 12 Gy volume and other (HI, CI) dosimetric parameters [Figure 3]. Hence, after planning the optimal plan, prior to approval there may be an option to consider beam reduction upto an extent without compromising on the dosimetric parameters.

\section{Impact of beam reduction \& optimization without changing the collimators in 12 Gy normal brain volume}

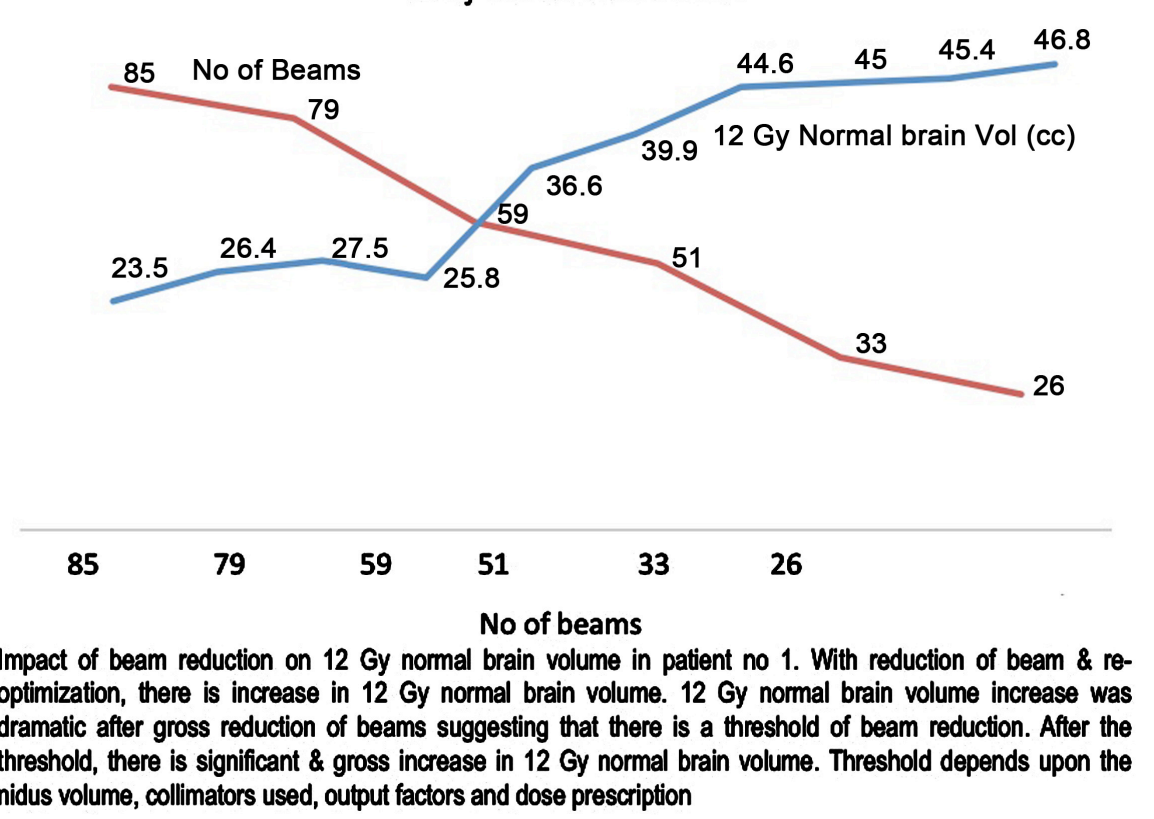

Figure 2. Changes in conformity with beamlet reduction. 
Beam reduction effect: Neurodeficit probability

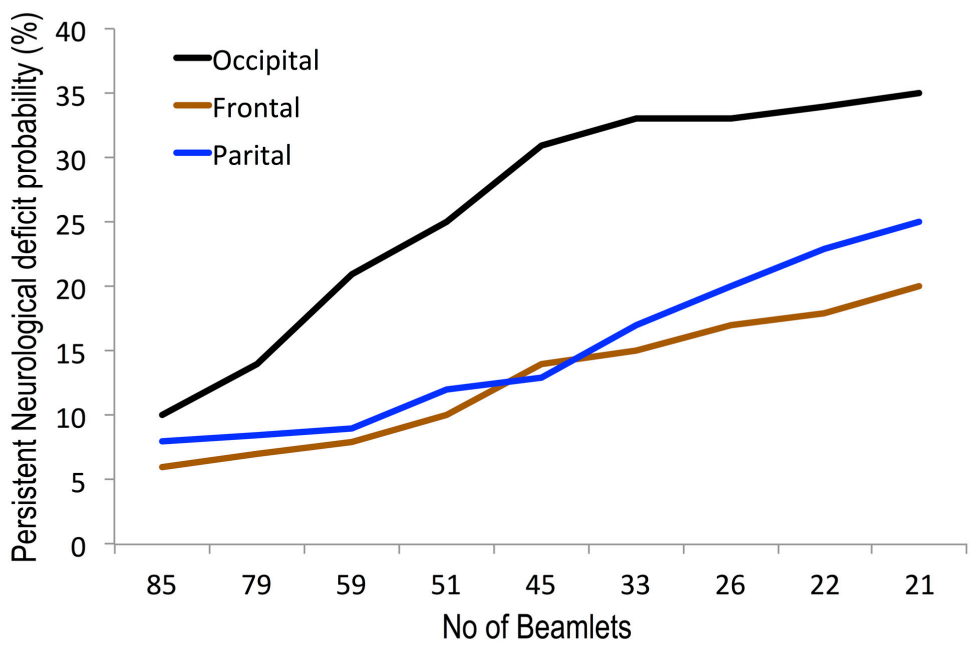

$20-50 \%$ increase in $\mathrm{PND}^{*}$ with beam reduction

*PND: Persistent Neurological deficit

Prediction done according to Flickinger et al. Rad Onc 2002; 63:347-354.

Figure 3. Effect of beamlet reduction on persistent neurological deficit.

\section{Discussion}

Arteriovenous malformation (AVM) is relatively uncommon but a unique lesion in brain. The outcome is excellent with surgery and radiosurgery [5] [6] [7] [8] [9]. Outcome (complete obliteration) depends upon the nidus volume, dose delivered, site of lesion, supplying and draining vessels and previous treatment. In suitable cases complete obliteration rate varies between $70 \%$ - 90\% depending upon various factors [10]. Majority of the patients present at young age or at adolecent. Rarely we do see elderly patients presenting with AVM. It is assumed that a proportion of "sudden death" with "unknown" etiology in adults may be related with bleeding from "occult" AVMs. Hence, though AVM is a benign condition, the implications are more serious and need interventions. Treatment with radiosurgery is not devoid of long-term complications. In a large series with long-term follow up, serious long term toxicities such as persistent neurological deficits are upto 9\% [5] [6] [7]. Toxicities after radiosurgery depend upon $12 \mathrm{~Gy}$ normal brain volume and site of lesion, as described by the Flickenger's model [5]. Nidus in eloquent areas of brain (thalamus, pons) has higher probability of long-term toxicity [6].

AVM radiosurgery was traditionally done with "gold standard" gammaknife radiosurgery system with invasive frame and multiple Co60 sources [13]. Usually from the available 201 non-coplanar cone source positions, suitable positions are selected to deliver dose to the nidus and prescription isodose was usually $50 \%$. Hence, there was inhomogeneity to the target and "hot spot" in the centre of the nidus with possibility of necrosis. In standard LA-based SRS systems [BrainLAB system], 5 to 9 fixed fields or limited number of arcs (2 to 6 arcs) were used with micro-MLC [14] [15]. There was significant improvement 
in target coverage and conformity with micro-MLCs; however some region of normal brain also received high dose especially in smaller targets [15]. Then, the "non-coplanar arc" based systems (arc based treatment delivery such as Brain$\mathrm{LAB}^{\circledast}$, RapidArc $\left.{ }^{\oplus}\right)$ came into practice [16]. “Arc" treatment basically consists of multiple beams in different coplanar or non-coplanar planes. Multiple beams in arc format improved conformity, and homogeniety and reduce dose spillage to normal brain region. Robotic radiosurgery with CyberKnife uses multiple nodes and beams to deliver the dose. Usually from available 1200 nodes, 100 to 200 nodal points are used to deliver dose in a non-coplanar manner [17] [18] [19]. Small cones used to deliver dosage ensure high conformity and minimal dose spillage. There is "intra-fraction" tracking and usually no or minimal PTV (ITV) margin with robotic radiosurgery system; hence the target volume (PTV) is relatively smaller compared with other systems required to give 1 to $2 \mathrm{~mm}$ PTV margin [11] [17]. "Multiple isocentres" do have advantage of reducing high dose region and improving homogeniety.

Delivery acuracy with robotic radiosurgery system is implicable. Also as there is no or minimal PTV margin required, target volume is smaller, but there are issues with compliance because of longer treatment time [12]. In Multiplan system, plan evaluation usually consists of target coverage, OAR dose parameters, prescription isodose and total monitor units. There is a need to evaluate the plan in respect of number of beams and treatment time as well [20]. Reduction of beams by reducing beams with lesser monitor unit contributions may alter the dose distribution parameters and reduce coverage. On the other hand, with reduction of beams with minimal contribution may not have significant changes in dosimetric parameters until a threshold is reached. Robotic radiosurgery plan may be optimized in terms of number of beams and treatment time, apart from the standard parameters. In the present study, when beam reduction protocol was applied without compromising the target coverage, there was no significant influence on HI, CI and also 12 Gy volume till a threshold was reached. After the threshold, with minimal reduction of beams, there was significant increase in 12 Gy normal brain volume and deterioration in both conformity and homogeniety indices.

In initial reduction of beams, there was only moderate increase in delivered monitor unit. Hence, initially even though there was reduction of beams, there was not significant deteriortaion in plan dosimetric parameters. Further reduction of beams with lesser monitor unit contribution, there was significant deteriortaion in dosimetric parameters. Beam reduction in multiple non-coplanar systems has a thereshold for beam reduction. Any attempt to reduce further beamlets will impair the plan dosimetry.

This phenomenon may be considered with another perspective as well. Fixed beams planused in earlier systems (eg. BrainLAB ${ }^{\circledR}$ ) and there are possibilites for improvement of coverage and reduction of 12 Gy normal brain volumes if planned with multiple non-coplanar beams. It can be assumed that multiple beams with robotic radiosurgery system or arc radiation therapy delivery sys- 
tems do have an advantage of reduction of 12 Gy normal brain volume and hence long-term toxicities. Reduction of beams in robotic radiosurgery system increase 12 Gy volume and there are proportional increase in the probability of late toxicities (persistent neurological deficits) [Figure 3] [10] [17]. Probability of increase in late toxicity with increase in 12 Gy normal brain volume was calculated as per the Flickenger's model. Probability of increase in late toxicity with increase in 12 Gy normal brain volume is higher in occipital region lesions than in parital or frontal lobe lesion.

It may be assumed that, with modern arc treatment or multiple beams there is a possibility of treatment of larger volume nidus without increasing 12 Gy volume and hence late toxicity. In the present study, analysis was done in $2.5 \mathrm{~cm}$, $3.5 \mathrm{~cm}$ and $4.5 \mathrm{~cm}$ diameter nidus in same region of brain; planned with similar marginal dose (20 Gy/1fr), coverage (98\%) and planning algorithm [17]. There were significant increases in 12 Gy volumes with increase in nidus volumes. Hence, even with modern multiple beam robotic radiosurgery system we are not able to treat larger volume nidus without increasing late toxicities. Modern radiosurgery delivery systems with arc or multiple non-coplanar beams are safer in small nidus compared with fixed field radiosurgery systems, however it may not be benificial in larger volume nidus to reduce toxicity [7] [17].

In summary, multiple beam robotic radiosurgery systems with non-coplanar beams may have dosimetric superiority compared with conventional fixed field radiosurgery systems. In robotic radiosurgery systems, there is a threshold for beam reduction. There is a need for adequate number of beams (threshold) to achieve optimal plan. The threshold depends upon the nidus volume, site of lesion and planning indices such as number of cons used, optimization algorithm and output parameters.

\section{Study Presented}

Present data was present at the Annual meeting of Indian Society of Neurooncology (ISNOCON), Lucknow India.

\section{Disclaimer}

None.

\section{Conflicts of Interest}

None.

\section{References}

[1] Touboul, E., Al Halabi, A. and Buffat, L. (1998) Single-Fraction Stereotactic Radiotherapy: A Dose-Response Analysis of Arteriovenous Malformation Obliteration. International Journal of Radiation Oncology Biology Physics, 41, 855-861. https://doi.org/10.1016/S0360-3016(98)00115-1

[2] Zabel, A., Milker-Zabel, S., Huber, P., et al. (2005) Treatment Outcome after LINAC-Based Radiosurgery in Cerebral Arteriovenous Malformations: Retrospec- 
tive Analysis of Factors Affecting Obliteration. Radiotherapy and Oncology, 77, 105-110. https://doi.org/10.1016/j.radonc.2005.04.008

[3] Pollock, B., Gorman, D. and Coffey, R. (2003) Patient Outcomes after Arteriovenous Malformation Radiosurgical Management: Results Based on a 5 to 14-Year Follow-Up Study. Journal of Neurosurgery, 52, 1291-1297. https://doi.org/10.1227/01.NEU.0000064800.26214.FE

[4] Flickinger, J., Kondziolka, D., Maitza, A. and Lunsford, L. (2002) An Analysis of the Dose-Response for Arteriovenous Malformation Radiosurgery and Other Factors Affecting Obliteration. Radiotherapy and Oncology, 63, 347-354. https://doi.org/10.1016/S0167-8140(02)00103-2

[5] Flickinger, J.C., Kondziolka, D., Lunsford, L.D., Kassam, A., Phuong, L.K., Liscak, R. and Pollock, B. (2000) Development of a Model to Predict Permanent Symptomatic Postradiosurgery Injury for Arteriovenous Malformation Patients. International Journal of Radiation Oncology Biology Physics, 46, 1143-1148. https://doi.org/10.1016/S0360-3016(99)00513-1

[6] Flickinger, J., Kondziolka, D., Maitz, A. and Lunsford, L. (1998) Analysis of Neurological Sequelae from Radiosurgery of Arteriovenous Malformations: How Location Affects Outcome. International Journal of Radiation Oncology Biology Physics, 40, 273-278. https://doi.org/10.1016/S0360-3016(97)00718-9

[7] Jalali, R., Dutta, D., Srinivas, C., Munshi, A., Limaye, U., Goel, A., Deshpande, D. and Sarin, R. (2009) Micromultileaf Collimator-Based Stereotactic Radiosurgery for Selected Arteriovenous Malformations: Technique and Preliminary Experience. Journal of Cancer Research and Therapeutics, 5, 186-191. https://doi.org/10.4103/0973-1482.57124

[8] Colombo, F., Cavedon, C., Casentini, L., Francescon, P., Causin, F. and Pinna, V. (2009) Early Results of CyberKnife Radiosurgery for Arteriovenous Malformations. Journal of Neurosurgery, 111, 807-819. https://doi.org/10.3171/2008.10.JNS08749

[9] Wowra, B., Muacevic, A., Tonn, J.C., Schoenberg, S.O., Reiser, M. and Herrmann, K.A. (2009) Obliteration Dynamics in Cerebral Arteriovenous Malformations Aftercyberknife Radiosurgery: Quantification with Sequential Nidus Volumetry and 3-Tesla 3-Dimensional Time-of-Flight Magnetic Resonance Angiography. Neurosurgery, 64, A102-A109. https://doi.org/10.1227/01.NEU.0000339201.31176.C9

[10] Yu, C., Jozsef, G., Apuzzo, M.L. and Petrovich, Z. (2003) Dosimetric Comparison of CyberKnife with Other Radiosurgical Modalities for an Ellipsoidal Target. Neurosurgery, 53, 1155-1162. https://doi.org/10.1227/01.NEU.0000088805.01793.5A

[11] Gevaert, T., Levivier, M., Lacornerie, T., Verellen, D., Engels, B., Reynaert, N., Tournel, K., Duchateau, M., Reynders, T., Depuydt, T., Collen, C., Lartigau, E. and De Ridder, M. (2013) Dosimetric Comparison of Different Treatment Modalities for Stereotactic Radiosurgery of Arteriovenous Malformations and Acoustic Neuromas. Radiotherapy and Oncology, 106, 192-197. https://doi.org/10.1016/j.radonc.2012.07.002

[12] Lomax, N.J. and Scheib, S.G. (2003) Quantifying the Degree of Conformity in Radiosurgery Treatment Planning. International Journal of Radiation Oncology Biology Physics, 55, 1409-1419. https://doi.org/10.1016/S0360-3016(02)04599-6

[13] Andrews, D., Bednarz, G., Evans, J. and Downes, B. (2006) A Review of 3 Current Radiosurgery Systems. Surgical Neurology, 66, 559-564. https://doi.org/10.1016/j.surneu.2006.08.002

[14] Blamek, S., Grządziel, A., Miszczyk, L., et al. (2013) Robotic Radiosurgery versus Micro-Multileaf Collimator: A Dosimetric Comparison for Large or Critically Lo- 
cated Arteriovenous Malformations. Radiation Oncology, 8, 205.

https://doi.org/10.1186/1748-717X-8-205

[15] Ohtakara, K., Hayashi, S., Tanaka, H. and Hoshi, H. (2011) Dosimetric Comparison of $2.5 \mathrm{~mm}$ vs. $3.0 \mathrm{~mm}$ Leaf Width Micro-Multileaf Collimator-Based Treatment Systems for Intracranial Stereotactic Radiosurgery Using Dynamic Conformal Arcs: Implications for Treatment Planning. Japanese Journal of Radiology, 29, 630-638. https://doi.org/10.1007/s11604-011-0606-6

[16] Colombo, F., Pozza, F., Chierego, G., Casentini, L., De Luca, G. and Francescon, P. (1994) Linear Accelerator Radiosurgery of Cerebral Arteriovenous Malformations: An Update. Neurosurgery, 34, 14-20. https://doi.org/10.1227/00006123-199401000-00004

[17] Friedman, W., Bova, F. and Mendenhall, W. (1995) Linear Accelerator Radiosurgery for Arteriovenous Malformations: The Relationship of Size to Outcome. Journal of Neurosurgery, 82, 180-189. https://doi.org/10.3171/jns.1995.82.2.0180

[18] Wagner, T.H., Bova, F.J., Friedman, W.A., Buatti, J.M., Bouchet, L.G. and Meeks, S.L. (2003) A Simple and Reliable Index for Scoring Rival Stereotactic Radiosurgery Plans. International Journal of Radiation Oncology Biology Physics, 57, 1141-1149. https://doi.org/10.1016/S0360-3016(03)01563-3

[19] Dutta, D., Balaji Subramanian, S., Murli, V., Sudahar, H., Gopalakrishna Kurup, P.G. and Potharaju, M. (2012) Dosimetry Comparison of Linac-Based (BrainLAB) and Robotic Radiosurgery (CyberKnife) Stereotactic System Plans for Acoustic Schwannoma. Journal of Neuro-Oncology, 106, 637-642. https://doi.org/10.1007/s11060-011-0703-5

[20] Laakso, A., Dashti, R., Seppänen, J., Juvela, S., Väärt, K., Niemelä, M., Sankila, R. and Hernesniemi, J.A. (2008) Long-Term Excess Mortality in 623 Patients with Brain Arteriovenous Malformations. Neurosurgery, 63, 244-253.

https://doi.org/10.1227/01.NEU.0000320439.27895.24 